\title{
BMJ Open Utilisation of healthcare services for respiratory tract infections in patients with and without diabetes in Qatar: a cross-sectional study
}

\author{
Nazmul Islam, ${ }^{1}$ Tawanda Chivese, ${ }^{2}$ M Fasihul Alam (D) ${ }^{1}$
}

To cite: Islam N, Chivese T, Alam MF. Utilisation of healthcare services for respiratory tract infections in patients with and without diabetes in Qatar: a crosssectional study. BMJ Open 2020;10:e041761. doi:10.1136/ bmjopen-2020-041761

- Prepublication history for this paper is available online. To view these files, please visit the journal online (http://dx.doi. org/10.1136/bmjopen-2020041761).

Received 16 June 2020

Revised 12 November 2020 Accepted 24 November 2020

D Check for updates

(C) Author(s) (or their employer(s)) 2020. Re-use permitted under CC BY-NC. No commercial re-use. See rights and permissions. Published by BMJ.

${ }^{1}$ Department of Public Health, College of Health Sciences, QU Health, Qatar University, Doha, Qatar

${ }^{2}$ Department of Population Medicine, College of Medicine, QU Health, Qatar University, Doha, Qatar

Correspondence to Dr M Fasihul Alam; malam@qu.edu.qa

\section{ABSTRACT}

Objective People with diabetes mellitus have a high risk of acquiring respiratory tract infections (RTIs), yet little is known about their utilisation of healthcare services compared with people without diabetes. This study aimed to compare the utilisation of healthcare services for RTls between individuals with and without diabetes attending primary healthcare centres (PHCCs) in Qatar.

Design A retrospective cross-sectional study was conducted using an electronic database of all individuals who had a diagnosis of RTI.

Setting PHCCs in Qatar from July 2015 to December 2017.

Participants Participants in the study were all adult individuals (aged $\geq 18$ years) who visited the primary healthcare facilities and were diagnosed with an RTI during the study period.

Primary and outcome measures For each participant, visits to the healthcare facility, antibiotic use and use of other medications were extracted from the electronic database and compared between participants with and without a diabetes diagnosis.

Results A total of 32857 participants were included, of whom $7407(22.5 \%)$ had a diabetes diagnosis. Results from a negative binomial regression indicate that diabetes diagnosis was significantly associated with increased visits to the healthcare facility (incidence rate ratio (IRR) $1.10,95 \% \mathrm{Cl} 1.076$ to $1.134, \mathrm{p}<0.001$ ), antibiotic use (IRR $1.09,95 \% \mathrm{Cl} 1.046$ to $1.145, \mathrm{p}<0.001)$ and use of other medications (IRR 1.11, $95 \% \mathrm{Cl} 1.078$ to $1.143, \mathrm{p}<0.001$ ). Conclusions A diabetes diagnosis among patients with RTI was associated with higher utilisation of healthcare services. Given the added costs to the healthcare system, prevention of diabetes will have additional benefits to the healthcare system, apart from diabetes-associated costs alone.

\section{INTRODUCTION}

The worldwide prevalence of diabetes mellitus (DM) has increased from $4.7 \%$ in 1980 to $8.8 \%$ in 2017 among adults (WHO). ${ }^{12}$ By the year 2045, the prevalence of diabetes is expected to increase to $9.9 \% .^{2}$ The Middle East and North African (MENA) region has the highest prevalence of diabetes $(12.8 \%)$, compared with other International Diabetes

\section{Strengths and limitations of this study}

- This is first study in the Middle East North Africa region, where research comparing the utilisation of healthcare services for respiratory tract infections (RTIs) by people with and those without diabetes are scarce.

- From a large cohort of 33000 participants, a diabetes diagnosis was associated with a higher risk of healthcare facility visits, higher use of antibiotics and a higher use of other medications, for RTI.

- People living with diabetes may require more healthcare services and this may need to be considered in healthcare financing.

- Some patients might have had undiagnosed diabetes diagnosis, and it was not possible to distinguish the effect by the type of diabetes.

- We were not able to capture some of the other (hospital) healthcare services used by patients with RTI due to the limitations of the underlying electronic database used.

Federation regions. ${ }^{3}$ In the absence of effective interventions and/or policies, the prevalence in the MENA region is projected to increase to $14.2 \%$ in 2030 and $15.7 \%$ in 2045 , while the number of people with diabetes is expected to increase to 55 million in 2019 and to 108 million in $2045 .{ }^{3}$ Qatar, situated in the MENA region, is one of the countries with a high prevalence of diabetes, estimated at $15.5 \%$ in $2019 .{ }^{3}$ Diabetes contributed $16.2 \%$ to all-cause mortality in the MENA region in 2019, and the region has the second-highest proportion of diabetes-related deaths in people under the age of 60 years, after Africa. ${ }^{3}$

Apart from its direct effect on morbidity and mortality, diabetes is associated with a higher risk of non-communicable diseases and infections. ${ }^{4}$ Because of diabetes-related impairments to the immune systems, people with diabetes are also at high risk of infectious diseases. ${ }^{5}$ Individuals living with diabetes are more susceptible to cellulitis, ${ }^{6}$ urinary tract 
infections, ${ }^{6}$ tuberculosis ${ }^{56}$ and other upper respiratory tract infections (RTIs). ${ }^{5}$ This increased risk of infection is due to inadequate control of serum blood glucose, diabetic neuropathy and reduced innate and adaptive immune responses. ${ }^{78}$ People with diabetes are also at greater risk for community-acquired infections and rare infections like malignant otitis externa, rhinocerebral mucormycosis and emphysematous pyelonephritis. ${ }^{7910}$ People with diabetes also have a higher risk for recurring infections or complications from infections, with the consequent need for frequent primary healthcare centre (PHCC) visits, antibiotic prescriptions, emergency visits and inpatient hospital management. ${ }^{6} 7$ Data from the ongoing COVID-19 pandemic caused by the SARS-CoV-2 show that people with diabetes are at high risk of both severe infection (pneumonia) and mortality from the disease. $^{11}$

On their own, RTIs impose a great burden on healthcare services. This burden is reflected by increased rates of consultations, antibiotic prescriptions, hospital admissions, length of stay and treatment costs for complications related to RTIs. ${ }^{6}$ For individuals with diabetes, RTIs can be a significant burden. The risk of a complicated course of lower RTI (LRTI) in individuals with diabetes has been reported to be almost twice as high as in the total elderly population. ${ }^{12}$ Several other studies have also shown that diabetes is associated with not only a higher risk of LRTIs, but is likely to have worse outcomes, including death. ${ }^{10}{ }^{13}$ Recent data form an observational study of nearly 3 million adults showed a twofold higher risk of death from COVID-19 infection in people with type 2 diabetes, which worsened with poor glycaemic control. ${ }^{13}$ This evidence suggests that when individuals with diabetes get infected with RTIs, they are likely to use additional healthcare services to manage their infections, compared with those without diabetes.

Although there is substantial evidence that diabetes is associated with an increased susceptibility to RTIs and worse outcomes, data on the utilisation of healthcare services by individuals with diabetes remain scarce. Limited research, mainly from Western Europe and North America, suggests that medical costs for individuals with diabetes are approximately 2.3 times higher than non-diabetic patients. ${ }^{7}$ Nearly half of the direct medical costs of diabetic care are believed to be associated with the management of diabetic complications. ${ }^{7}$ To our knowledge, there is no research that has assessed the utilisation of healthcare services in the MENA region. Given the increase in both the prevalence and the number of people with diabetes in the MENA region, there is a need to quantify the utilisation of healthcare services by people with diabetes. This study compares the use of primary healthcare services for RTIs between participants with and without diabetes. The services assessed in this study were RTI-related visits to the healthcare facility, use of antibiotics and use of other medication among patients attending PHCCs in Qatar.

\section{METHODS}

\section{Study design and population}

A retrospective cross-sectional study was conducted using patient-level electronic data for patients with RTI at seven PHCCs in Doha and other municipalities in Qatar. Eligible participants were patients aged 18 years and above attending selected PHCCs for RTIs between 1 July 2015 and 31 December 2017. Pregnant women were excluded.

\section{Healthcare system in Qatar}

Qatar's healthcare system, which can be broadly categorised into a public and a private healthcare system, is controlled by the Ministry of Public Health (MoPH), previously known as Supreme Council of Health. The MoPH does not deliver direct healthcare services, rather this is the responsibility of public health institutes, such as the Primary Health Care Corporation, the Hamad Medical Corporation and other private healthcare providers. There are some specialised centres as well such as Sidra Medicine and National Center for Cancer Care \& Research. Qatari citizens are covered by a national health insurance system, while expatriates must either receive health insurance from their employers or in the case of the self-employed, purchase insurance. ${ }^{14}$

The State of Qatar took its first step in establishing a primary healthcare system and started to deliver healthcare services through a variety of clinics as early as 1954 . In the year 1978, the Ministry of Health established a comprehensive structure for building a primary healthcare system which was submitted to the council of ministers and the system included the launching of primary healthcare services through nine health centres, covering different parts of the state, and capable of delivering basic and critical health and medical services. At present, the Primary Health Care Corporation has 23 PHCCs. Thirteen of these centres are located in Doha city, while the rest of the centres are located in other parts of the country. Many PHCCs also provide specialty services to the community aiming to enhance self-care model as well as healthy life style manner. Qataris have access to any PHCCs despite their home location but expatriates can access only PHCCs in which they are registered based on their home location. The 7 PHCCs included in this study were the largest of the 23 PHCCs and were spreading over different parts of the country. ${ }^{145}$

\section{Respiratory tract infections}

Diagnosis of RTIs was done by either clinical assessment or diagnostic tests. Patients presenting with the following: dry and wet cough, body malaise, runny nose, fever, sore throat and headache were clinically assessed and in some cases had diagnostic tests done. The diagnostic test included chest X-ray and mouth, nose and throat swabs for infective organisms. RTI diagnosis included both upper RTI (acute tonsillitis, pharyngitis, laryngitis, otitis media) and lower RTI (acute bronchitis, pneumonia and whooping cough). 


\section{Diabetes diagnosis}

Participants with a diabetes diagnosis were identified from the electronic database. Diabetes diagnosis was done using 2006 WHO guidelines. ${ }^{16}$ This included persons with different forms of diabetes including type 1 and type 2 diabetes. Similar to other countries, type 2 diabetes is the most common form of diabetes and is diagnosed according to the WHO diagnosis criteria of $2006 .{ }^{17}$ This usually includes people with clinical symptoms of hyperglycaemic, and/or fasting blood glucose $\geq 7 \mathrm{mmol} / \mathrm{L}$, and/or oral glucose tolerance test-2-hour blood glucose $\geq 11.1 \mathrm{mmol} / \mathrm{L}$-and/or hemoglobin A1c (HbA1c) $\geq 6.5 \%$, after ruling out other forms of diabetes. All patients with diabetes in this study were on treatment.

\section{Healthcare services utilisation}

The following health services were assessed for each participant: the total number of visits done by each participant for the RTI, total count of antibiotics pills prescribed for RTI and the total number of medications except antibiotics prescribed for RTI.

\section{Other data measured}

Other data measured included age in years, gender and data on other comorbidities which were extracted from the hospital records in the Cerner system. These comorbidities included obesity (defined at a body mass index above $30 \mathrm{~kg} / \mathrm{m}^{2}$ ) and any existing diagnoses of hypertension and dyslipidaemia.

\section{Sampling and sample size}

Every eligible patient who visited the primary care centres during the study period was included in the study. Data were retrieved from the Cerner electronic system. ${ }^{18}$ Although there are in total 23 PHCCs in Qatar, during the data analysis stage, the Cerner database had complete patient-level information for only seven PHCCs over the study period. Data from all eligible patients from these 7 centres were included in the analysis.

\section{Data extraction}

The Cerner electronic database provides patient-level information on age, gender, comorbidity status, RTIrelated General Practioner (GP) visits at PHCCs, antibiotics and other medications use. The recorded diagnosis in the database was considered as final and not verified externally. Duplicate participants were excluded. Data were validated by the PHCG validation team by taking random samples from each month and checking these against paper records. The RTI diagnoses for patients are based on the standard codes using the International Classification of Diseases, 10th Revision.

\section{Statistical analysis}

Baseline and outcome data were presented as frequency and percentages for categorical variables, and for numerical variables, they were presented as means and SDs and median and IQR for non-normally distributed variables, as appropriate. In some cases, both mean (SD) and median (IQR) are reported to enable comparisons with other studies. Data on hospital visits, antibiotic pill counts and pill counts of other medications were not normally distributed but the mean (SD) is reported to illustrate differences between participants with and without diabetes. Comparisons between participants with and without diabetes were done using $\chi^{2}$ tests for categorical variables. Student's t-tests were used for normally numerical data and Wilcoxon rank-sum tests were used to compare groups for non-normally distributed numerical data.

Given individual healthcare service utilisation data were available at primary care centre level, an investigation of centre-level variation is deemed to be appropriate. However, the healthcare system allows a Qatari citizen to visit any health centre for healthcare services. This means, an individual can be linked with different healthcare centres, which makes it difficult to examine centre-level effects on the utilisation using patient-level data. A crude analysis involving multilevel modelling framework did not show any significant centre-level variation in service utilisation, hence further analyses did not estimate a centre-level effect.

To investigate the association between diabetes diagnosis and utilisation of each of the healthcare services, a multiple variable negative binomial regression model was estimated, after adjusting for a number of demographic and comorbidity factors. The negative binomial model was chosen as the outcome variables were counts and overdispersed. If data appear to be not overdispersed, a Poisson regression could be estimated. We have assessed the overdispersion of data by considering a null hypothesis of $\alpha$ (overdispersion parameter) $=0$, which is tested using a likelihood ratio (LR) $\chi^{2}$ test. Regression coefficients of the negative binomial model parameters were interpreted as the difference between the log of expected counts or incidence rate ratios (IRRs). All statistical analyses were two-sided, and a p- value less than 0.05 was considered as statistically significant. Analyses were done using the Stata statistical software package, and 15\% and 95\% CIs were reported for estimates, where appropriate.

\section{Patient and public involvement}

Patients and the public were not involved in the design, conduct or reporting of this research.

\section{RESULTS}

\section{Characteristics of participants}

A total of 32857 participants were included, of whom 7407 $(22.5 \%)$ had a diabetes diagnosis. Participants with diabetes were significantly older than participants without diabetes (mean (SD) 51.4 (15.4) vs 33.2 (13.0) years, respectively, $\mathrm{p}<0.001)$. The proportion of females was significantly higher in participants with diabetes, compared with those without $(61.6 \%$ vs $51.5 \%, \mathrm{p}<0.001)$. Compared with participants without diabetes, there were higher proportions of comorbidities in participants with diabetes (table 1). 
Table 1 Characteristics of participants and comparison by diabetes diagnosis status

\begin{tabular}{|c|c|c|c|c|c|}
\hline Variables & Level & Overall, $\mathrm{N}=32857$ & Diabetes, $\mathrm{N}=7407$ & No diabetes, $\mathrm{N}=25450$ & P value \\
\hline \multicolumn{6}{|l|}{ Age category } \\
\hline & $30-49$ years & $12032(36.6)$ & $2422(32.7)$ & $9610(37.8)$ & \\
\hline & $\geq 50$ years & 7513 (22.9) & $4236(57.2)$ & 3277 (12.9) & \\
\hline & Male & $15199(46.3)$ & $2843(38.4)$ & $12356(48.6)$ & $<0.001$ \\
\hline & Female & $17658(53.7)$ & $4564(61.6)$ & $13094(51.5)$ & \\
\hline Hypertension, n (\%) & Yes & 6187 (18.8) & $4113(55.5)$ & 2074 (8.2) & $<0.001$ \\
\hline Asthma, n (\%) & Yes & $4268(13.0)$ & $1588(21.4)$ & $2680(10.5)$ & $<0.001$ \\
\hline Dyslipidaemia, n (\%) & Yes & 3480 (10.6) & 2584 (35.0) & $896(3.5)$ & $<0.001$ \\
\hline
\end{tabular}

${ }^{*} \mathrm{P}$ values are based on t-test, $\chi^{2}$ statistic and Wilcoxon rank-sum tests.

†Percentages are column percentages.

\section{Healthcare services utilisation}

Table 2 shows a comparison of healthcare services utilisation between participants with and those without a diabetes diagnosis. Participants with diabetes had a significantly higher total number of visits due to RTI than participants with no diabetes (mean 3.08 (SD 3.23) vs 2.63 (SD 2.70), respectively, $\mathrm{p}<0.001)$. Further, participants with a diabetes had significantly higher antibiotic pill counts compared with those without a diagnosis of diabetes (mean 0.88 (SD 1.3 ) vs 0.80 (SD 1.2), respectively, $\mathrm{p}<0.001$ ), and had significantly higher number of other medications that they were taking (mean 8.0 (SD 8.9) vs 6.7 (SD 7.7), respectively, $\mathrm{p}<0.001)$.

\section{Association between diabetes diagnosis and healthcare services utilisation}

$\chi^{2}$ values from an LR Test reject the null hypothesis that the overdispersion parameter $\alpha=0$ (table 3), suggesting a negative binomial distribution fits data well. However, for sensitivity analysis, a Poisson regression model was also estimated, which produced almost similar results (not presented).
Estimates from a negative binomial multivariate regression suggest that a diabetes diagnosis in participants was significantly associated with more visits (10\% increase) to the health facility (IRR $1.10,95 \%$ CI 1.076 to 1.134 , $\mathrm{p}<0.001$ ), more antibiotic pill counts (9\% increase) (IRR $1.09,95 \%$ CI 1.046 to $1.145, \mathrm{p}<0.001)$ and higher pill counts of other medications (11\% increase) (IRR $1.11,95 \% \mathrm{CI}$ 1.078 to $1.143, \mathrm{p}<0.001)$, compared with those without a diabetes diagnosis (table 3 ).

Age, gender, hypertension, dyslipidaemia and obesity were also highly significantly associated with healthcare services utilisation (table 3).

\section{DISCUSSION}

In this study, we have demonstrated that individuals diagnosed with diabetes, compared with those without diabetes, are likely to use more health services associated with higher use of other healthcare services when they have RTIs. To our knowledge, this is the first ever study to report data on utilisation of healthcare services by people with diabetes in

\begin{tabular}{llccc}
\hline $\begin{array}{l}\text { Table } 2 \\
\text { diabetes }\end{array}$ & Comparison of healthcare service utilisation for respiratory tract infection between participants with and without \\
\hline Healthcare service & & $\begin{array}{l}\text { Diabetes, } \\
\mathbf{n = 7 4 0 7}\end{array}$ & $\begin{array}{l}\text { No diabetes, } \\
\mathbf{n = 2 5 4 5 0}\end{array}$ & P value $^{*}$ \\
\hline Total visits & Mean (SD) & $3.08(3.23)$ & $2.63(2.70)$ & $<0.001$ \\
Total visits & Median (IQR) & $2.00(1.00-4.00)$ & $2.00(1.00-3.00)$ & $<0.001$ \\
\hline Total antibiotic pill count & Mean (SD) & $0.88(1.31)$ & $0.80(1.21)$ & $<0.001$ \\
Antibiotics & Median (IQR) & $0.00(0.00-1.00)$ & $0.00(0.00-1.00)$ & $<0.001$ \\
Other medications pill count & Yes, $\mathrm{n}(\%)$ & $3589(48.45)$ & $11924(46.85)$ & 0.015 \\
Other medications pill count & Mean (SD) & $8.03(8.87)$ & $6.96(7.72)$ & $<0.001$ \\
\hline
\end{tabular}

${ }^{*} \mathrm{P}$ values are based on t-test and Wilcoxon rank-sum tests. 
Table 3 Association between a diabetes diagnosis and utilisation of healthcare services-multiple variable negative binomial regression

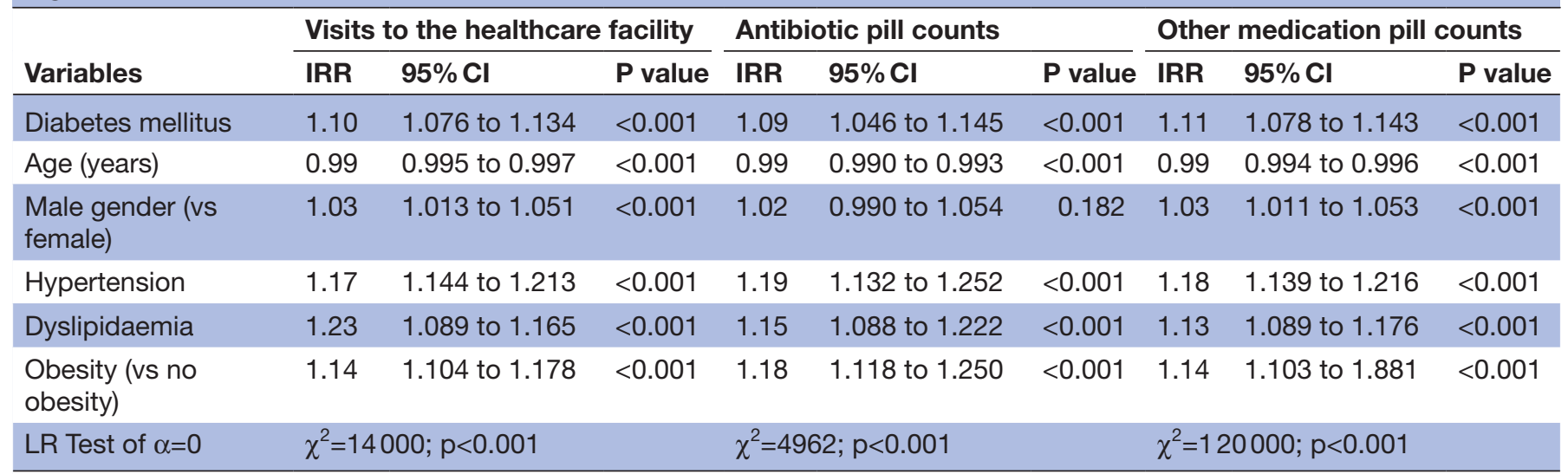

$\alpha$-overdispersion parameter.

$\mathrm{IRR}$, incidence rate ratio; LR, likelihood ratio.

the MENA, a region with the highest prevalence of diabetes in the world. During this study of 32857 participants, we found that when participants with a diabetes diagnosis had an RTI, they made more visits to the healthcare facilities, used more antibiotics and were likely to be taking other medications, compared with those without a diabetes diagnosis. A diabetes diagnosis remained strongly associated with higher use of the healthcare services after adjusting for age, gender and other comorbidities.

We found that participants with diabetes had a significantly higher total number of PHCC visits due to RTI than participants with no diabetes. These findings are consistent with the few studies that have reported either higher numbers of hospital visits or higher rates of hospitalisations in Australia, ${ }^{19}$ the $\mathrm{USA}^{7}$ and Canada. ${ }^{9}$ For example, Korbel et al found that, in the USA, individuals with diabetes frequently went to emergency departments for care, were routinely hospitalised because of infectious diseases and incurred high hospital charges. ${ }^{7}$ There are multiple reasons behind this. First, having an infection can worsen blood glucose metabolism and result in the need for hospitalisation. ${ }^{20}$ Second, high blood glucose levels weaken the individual immune system defences and make them vulnerable to infection. ${ }^{21}$ Therefore, individuals with diabetes are more prone to severe infections, recurrent infections, rare infections and are more likely to develop complications related to infections, which require more medical treatment. ${ }^{22}$ Third, acute care clinicians are more likely to refer a patient who has diabetes for hospital admissions. This practice may result in a patient being hospitalised with an infection that could be adequately treated in a primary care facility without the need for admission. ${ }^{9}$ One way to reduce the need for frequent hospital visits and hospitalisation is adequate control of blood glucose. However, there is a paucity of research on the effect of blood glucose control on utilisation of healthcare services in the MENA region. There is also a need for clinical guidelines to include RTIs as a complication of diabetes and encourage clinicians to routinely screen for RTIs in people with diabetes.
Nevertheless, the consistency in findings from the study and those from the above literature ${ }^{7919}$ must also be interpreted carefully, partly because healthcare systems and healthcare service provisions across different countries vary. For example, in Australia and the USA, healthcare systems are mainly private health insurance based, unlike one in Qatar where there is a combination of both public and private healthcare providers for Qatari population. In Canada, it is predominantly social insurance-based publicly funded healthcare providers. This difference in systems and service provisions might have differential impact on individual-level healthcare utilisation.

Our findings contribute to the current debate on the effect of diabetes on the severity of COVID-19. ${ }^{23}$ Data from China and Europe have shown that people with diabetes are at high risk of COVID-19 and have a high risk of mortality from the disease. ${ }^{2425}$ The high risk for both infection and worse prognosis from infection has also been reported in studies of SARS in $2002^{26} 27$ and the Middle Eastern respiratory syndrome in $2012 .{ }^{5}$ In the current COVID-19 pandemic, individuals with diabetes have been found to have a higher risk for severe infection even when compared with those with cardiovascular disease and cancer. ${ }^{25}$ Projections are that respiratory pandemics will occur more frequently in the future,${ }^{28}$ and people with diabetes will remain at high risk of adverse outcomes. Our findings show that, even outside of the pandemics, people with diabetes require more healthcare resources. Up to $90 \%$ of people with diabetes have type 2 diabetes mellitus, ${ }^{3}$ which is preventable. Therefore, the prevention of diabetes is important, not only in reducing health expenditure but will also decrease the proportion of the population that is vulnerable to respiratory infection pandemics.

We found that participants with a diagnosis of diabetes had a significantly higher antibiotics pill count compared with those without a diagnosis of diabetes. Our findings are in agreement with research from the USA, ${ }^{21}$ where people with diabetes had a higher number of antibiotic 
prescriptions, compared with age-matched controls. Similar findings were also reported in Denmark. ${ }^{29}$ One immediate drawback of the higher use of antibiotics in people with diabetes is the high risk of antibiotic drug resistance. ${ }^{30}$ We did not assess whether the use of antibiotics was justified or not, and there is a need of further research for this in Qatar and the MENA region.

We also found that participants with a diagnosis of DM had significantly received more medications. This could be because people with diabetes are likely to develop other abnormalities related to DM such as microvascular complications (such as neuropathy, nephropathy and retinopathy), macrovascular complications (such as peripheral arterial disease, coronary artery disease and cerebrovascular disease) and altered dyslipidaemia. ${ }^{21} 31$ However, using more medications may lead to medication non-adherence, ${ }^{31}$ therefore clinicians may need to consider ways to reduce the total amounts of medications prescribed to patients.

We also found a strong association between hypertension, dyslipidaemia, obesity and use of healthcare services. These conditions, together with diabetes, have common physiological and environmental causes ${ }^{32}$ and insulin resistance is believed to play a central role. ${ }^{33}$ Obesity is associated with chronic insulin resistance, possibly through central adiposity-induced chronic inflammation in the liver. ${ }^{34}$ Notably, central adiposity is also the strongest cause of type 2 diabetes. ${ }^{33}{ }^{34}$ Our findings show that these cardiovascular factors are all associated with increased usage of healthcare services and suggest that a holistic approach for preventing them will have multiple benefits.

Our study adds to the understudied area of utilisation of healthcare services for RTIs by individuals with diabetes at primary healthcare facilities. By using a sufficiently large sample size, we were able to demonstrate that, during routine care, individuals with diabetes are likely to use more healthcare services in primary care settings. However, our study has several limitations. Due to the use of routine data, it is possible that some participants with diabetes may not have been diagnosed, although this is mitigated by the fact that the state of Qatar has a low prevalence of undiagnosed diabetes. Further, the Cerner database, which was the source of our data, does not include information on hospital-level healthcare services used by patients, and has no linkage to track referred cases, which limited our analysis and we could not assess other secondary services that the participants may have used. Again due to limitations of the data available from the Cerner system, we were also not able to analyse the effect of the type of diabetes or different severity levels of diabetes, although we assumed the majority of the participants had type 2 diabetes. Therefore, our findings are likely to be generalisable to individuals with type 2 diabetes. Further, we also had no data on the type of therapy that the participants were on, and could not assess this effect. Furthermore, all outcomes were derived from routine data collected from the electronic database of the PHCC, and the quality of these data was beyond the control of the researchers. Another limitation of our current analysis is that the database does not include many patient-level socioeconomic variables, such as body mass index (BMI) and income, potential confounders that we could have adjusted for in our analyses. In addition, although it is not likely, it is possible that the different primary care centres may have different prescribing patterns and that antibiotics and medications prescribed among the seven centres are not comparable. The lack of individual drug and dosage information for each patient is a limitation of the current study which may need to be addressed in future studies. Lastly, due to the nature of the healthcare system where Qatari citizen can visit any primary healthcare centre for services, an investigation of centre-level variation using individual patientlevel data was not possible.

\section{CONCLUSIONS}

A diabetes diagnosis is associated with higher use of primary healthcare services, particularly visits to the healthcare facility, use of antibiotics and use of other medications in Qatar. Given the added costs to the healthcare system, prevention of diabetes will have additional benefits to the healthcare system, apart from a reduction in morbidity, mortality and costs associated diabetes alone.

Acknowledgements The authors greatly acknowledge the support from the Primary Health Care Corporation in Qatar for providing necessary data for the study. We are also grateful to the Department of Public Health, Qatar University, faculties for their valuable comments and suggestions on the initial draft of the proposal.

Contributors MFA (senior and corresponding author) conceived and led the study plan and design. NI and MFA contributed to data collection, statistical data analyses and initial draft of the manuscript. $\mathrm{NI}$ and TC contributed to conception of, data analysis and writing up of the revised version of the manuscript. All authors provided a critical review, approved the final submission and agreed to be accountable for all aspects of the manuscript. MFA is the guarantor of the manuscript.

Funding Part of the study was funded by the Qatar University internal student grant no. QUST-1-CHS-2018-5 in terms of purchasing a software (Stata) for data analysis.

Competing interests None declared.

Patient consent for publication Not required.

Ethics approval The study received ethics approval and waiver of informed consent from the primary healthcare centre (PHCC) (Ref No: PHCC/RS/17/10/013) and the Qatar University Institutional Review Board (IRB) (Ref No: QU-IRB 875-E/18). All data were deidentified before analysis, and any personally identifying details were removed.

Provenance and peer review Not commissioned; externally peer reviewed.

Data availability statement All data analysed during this study are present in the manuscript. No additional data are available. Supporting data can be accessed via the corresponding author upon reasonable request.

Open access This is an open access article distributed in accordance with the Creative Commons Attribution Non Commercial (CC BY-NC 4.0) license, which permits others to distribute, remix, adapt, build upon this work non-commercially, and license their derivative works on different terms, provided the original work is properly cited, appropriate credit is given, any changes made indicated, and the use is non-commercial. See: http://creativecommons.org/licenses/by-nc/4.0/.

ORCID iD

M Fasihul Alam http://orcid.org/0000-0003-2590-851X 


\section{REFERENCES}

1 World Health O. Key facts, diabetes. World Health organization. Available: https://www.who.int/news-room/fact-sheets/detail/ diabetes

2 Cho NH, Shaw JE, Karuranga S, et al. IDF diabetes atlas: global estimates of diabetes prevalence for 2017 and projections for 2045. Diabetes Res Clin Pract 2018;138:271-81.

3 International Diabetes F. IDF diabetes atlas ninth edition 20192019.

4 Glovaci D, Fan W, Wong ND. Epidemiology of diabetes mellitus and cardiovascular disease. Curr Cardiol Rep 2019;21:21.

5 Morra ME, Van Thanh L, Kamel MG, et al. Clinical outcomes of current medical approaches for middle East respiratory syndrome: a systematic review and meta-analysis. Rev Med Virol 2018;28:e1977.

6 Pearson-Stuttard J, Blundell S, Harris T, et al. Diabetes and infection: assessing the association with glycaemic control in populationbased studies. Lancet Diabetes Endocrinol 2016;4:148-58.

7 Korbel L, Spencer JD. Diabetes mellitus and infection: an evaluation of hospital utilization and management costs in the United States. J Diabetes Complications 2015;29:192-5.

8 Chen SL, Jackson SL, Boyko EJ. Diabetes mellitus and urinary tract infection: epidemiology, pathogenesis and proposed studies in animal models. J Urol 2009;182:S51-6.

9 Shah BR, Hux JE. Quantifying the risk of infectious diseases for people with diabetes. Diabetes Care 2003;26:510-3.

10 Muller LMAJ, Gorter KJ, Hak E, et al. Increased risk of common infections in patients with type 1 and type 2 diabetes mellitus. Clin Infect Dis 2005;41:281-8.

11 Guo W, Li M, Dong Y, et al. Diabetes is a risk factor for the progression and prognosis of COVID -19. Diabetes Metab Res Rev 2020;36.

12 Bont J, Hak E, Hoes AW, et al. A prediction rule for elderly primarycare patients with lower respiratory tract infections. Eur Respir J 2007;29:969-75.

13 Holman N, Knighton P, Kar P, et al. Risk factors for COVID-19-related mortality in people with type 1 and type 2 diabetes in England: a population-based cohort study. Lancet Diabetes Endocrinol 2020;8:823-33.

14 MOPH Q, Health MoP. Health system Qatar. Available: https://www. moph.gov.qa/health-services/services-to-public/qatar-health-system

15 Corporation PHC. PHCC corporate profile. Available: https://www. phcc.qa/portal_new/index/index.php?limit=profile

16 World Health O. Definition and diagnosis of diabetes mellitus and intermediate hyperglycaemia: report of a WHO/IDF consultation 2006.

17 World Health O. Prevention of blindness from diabetes mellitus: report of a who consultation in Geneva, Switzerland, 9-11 November 2005. World Health Organization, 2006.
18 Khamis I. Electronic patient record system in Hamad medical Corporation, Qatar: challenges and improvements. Cybrarians Journal 2017

19 Hamilton EJ, Martin N, Makepeace A, et al. Incidence and predictors of hospitalization for bacterial infection in community-based patients with type 2 diabetes: the Fremantle diabetes study. PLoS One 2013;8:e60502.

20 Encinosa WE, Bernard D, Dor A. Does prescription drug adherence reduce hospitalizations and costs? the case of diabetes. Adv Health Econ Health Serv Res 2010;22:151-73.

21 Carey IM, Critchley JA, DeWilde S, et al. Risk of infection in type 1 and type 2 diabetes compared with the general population: a matched cohort study. Diabetes Care 2018;41:513-21.

22 Joshi N, Caputo GM, Weitekamp MR, et al. Infections in patients with diabetes mellitus. N Engl J Med 1999;341:1906-12.

23 The management of blood glucose should be Emphasized in the treatment of COVID-19. Journal of Sichuan University Medical Science 2020;51:146-50.

24 Goeijenbier M, van Sloten TT, Slobbe L, et al. Benefits of flu vaccination for persons with diabetes mellitus: a review. Vaccine 2017:35:5095-101.

25 Wu H, Lau ESH MRCW, et al. Secular trends in all-cause and cause-specific mortality rates in people with diabetes in Hong Kong, 2001-2016: a retrospective cohort study. Diabetologia 2020:1-10.

26 Huang Y-T, Lee Y-C, Hsiao C-J. Hospitalization for ambulatorycare-sensitive conditions in Taiwan following the SARS outbreak: a population-based interrupted time series study. J Formos Med Assoc 2009;108:386-94.

27 Chan-Yeung M, XU R-H. Sars: epidemiology. Respirology 2003;8:S9-14.

28 Daszak P, Olival KJ, Li H. A strategy to prevent future pandemics similar to the 2019-nCoV outbreak. Elsevier, 2020.

29 Mor A, Berencsi K, Nielsen JS, et al. Rates of community-based antibiotic prescriptions and Hospital-treated infections in individuals with and without type 2 diabetes: a Danish nationwide cohort study, 2004-2012. Clin Infect Dis 2016;63:501-11.

30 Carlet J, Jarlier V, Harbarth S, et al. Ready for a world without antibiotics? the pensières antibiotic resistance call to action. BioMed Central 2012.

31 Peron EP, Ogbonna KC, Donohoe KL. Antidiabetic medications and polypharmacy. Clin Geriatr Med 2015;31:17-27.

32 Stern MP. Diabetes and Cardiovascular Disease: The "Common Soil" Hypothesis. Diabetes 1995;44:369-74.

33 Lebovitz HE. Insulin resistance--a common link between type 2 diabetes and cardiovascular disease. Diabetes Obes Metab 2006;8:237-49.

34 Ye J. Mechanisms of insulin resistance in obesity. Front Med 2013;7:14-24. 